Article

\title{
Chassis Influence on the Exposure Assessment of a Compact EV during WPT Recharging Operations
}

\author{
Valerio De Santis ${ }^{1, *(1)}$, Luca Giaccone ${ }^{2}\left(\mathbb{D}\right.$ and Fabio Freschi ${ }^{2}(\mathbb{C}$ \\ 1 Department of Industrial and Information Engineering and Economics, University of L'Aquila, \\ 67100 L'Aquila, Italy \\ 2 Dipartimento Energia “G. Ferraris”, Politecnico di Torino, Corso Duca degli Abruzzi, 24, 10129 Torino, Italy; \\ luca.giaccone@polito.it (L.G.); fabio.freschi@polito.it (F.F.) \\ * Correspondence: valerio.desantis@univaq.it
}

Citation: De Santis, V.; Giaccone, L.; Freschi, F. Chassis Influence on the Exposure Assessment of a Compact EV during WPT Recharging

Operations. Magnetochemistry 2021, 7,

25. https://doi.org/10.3390/

magnetochemistry7020025

Academic Editor: Jamie L. Manson

Received: 9 January 2021

Accepted: 2 February 2021

Published: 7 February 2021

Publisher's Note: MDPI stays neutral with regard to jurisdictional claims in published maps and institutional affiliations.

Copyright: (c) 2021 by the authors. Licensee MDPI, Basel, Switzerland. This article is an open access article distributed under the terms and conditions of the Creative Commons Attribution (CC BY) license (https:// creativecommons.org/licenses/by/ $4.0 /)$.

\begin{abstract}
In this study, the external magnetic field emitted by a wireless power transfer (WPT) system and the internal electric field induced in human body models during recharging operations of a compact electric vehicle (EV) are evaluated. The magneticfield is calculated with a hybrid scheme coupling the boundary element method with the surface impedance boundary conditions in order to fit the multiscale open-boundary characteristics of the problem. A commercial software is then used to perform numerical dosimetry. Specifically, two realistic anatomical models, both in a driving position and in a standing posture, are considered, and the chassis of the EV is modeled either as a currently employed aluminum alloy and as a futuristic carbon fiber composite panel. Aligned and misaligned coil configurations of the WPT system are considered as well. The analysis of the obtained results shows that the International Commission on Non-Ionizing Radiation Protection (ICNIRP) reference levels are exceeded in the driving position, especially for the carbon fiber chassis, whereas the system is compliant with the basic restrictions, at least for the considered scenarios.
\end{abstract}

Keywords: computational electromagnetics; electric vehicle; EMF safety; low frequency dosimetry; wireless charging; wireless power transfer

\section{Introduction}

Air quality is becoming a health problem in many cities around the world. This is mainly due to the transportation sector, which is one of the main contributors towards global climate change and $\mathrm{CO}_{2}$ emissions [1]. Thus, the usage of compact electric vehicles (EVs), together with the development of new mobility services, is the most sustainable solution, at least in urban areas. However, despite having a wide appeal, EV deployment on a global scale is still hampered by the battery technology and charging infrastructure [2]. These drawbacks can be resolved through static or dynamic wireless power transfer (WPT) systems and their widespread applications in an improved charging infrastructure for EVs [3].

Another challenge pertinent to compact EVs is related to the choice of the chassis materials. Indeed, small EVs have to combine both mechanical strength, since they are seen as unsafe vehicles by most of the users, and light weight to extend battery life or increase vehicle performances. Current materials employed for the car body are aluminum (Al) alloys, but in the future, advanced lightweight materials, such as carbon fiber (CF) composite panels, could be widely used [4]. If, on the one hand, the latter can reduce the weight and the emission of the vehicles, on the other hand, the reduced conductivities of CF materials, which yield to lower shielding capabilities, arise more concerns about the EMF safety of passengers and bystanders.

This paper therefore deals with the evaluation of the human exposure to the magnetic field emitted by a WPT system aimed to recharge the battery of a compact car, namely a FIAT 500, when both Al and CF chassis are considered. The standalone design of the 
recharging system, which is a stationary WPT system operating at the nominal frequency of $85 \mathrm{kHz}$, can be easily performed with classical approaches, such as finite elements. However, when dealing with human exposure, the influence of the car body and the human body must also be taken into account [5-8]. It is well known that, within the low frequency (LF) range, the presence of the human body does not affect the source field. Therefore, it is possible to separate the complete analysis into two steps [9,10]: (1) simulation of the magnetic field source (WPT system and car body) and (2) numerical dosimetry (human body subject to the previously evaluated LF field).

The aforementioned steps are not trivial tasks. Indeed, the study of the magnetic field source is complicated by the presence of the car body, which is a large 3D surface with very small thickness. In this context, standard volume-based methods, like finite elements, are unaffordable in terms of mesh size and thus memory storage. Moreover, the reduced conductivities of CF materials make the solver even slower to converge, with longer simulation times. Thus, to reduce the computational burden, a hybrid approach based on the boundary element method (BEM) and surface impedance boundary conditions (SIBCs) has been adopted [11].

Numerical dosimetry in the LF range is commonly performed using the scalar potential finite difference (SPFD) formulation [9]. In this paper, the commercial software Sim4Life (https:/ / zmt.swiss/sim4life accessed on 7 February 2021) is employed. It makes use of the magnetic vector potential at the right-hand side that is not readily accessible from the hybrid formulation described earlier. For this reason, a curl-inversion procedure is used to compute a compatible magnetic vector potential starting from the magnetic flux density [12-14]. Sim4Life allowed us the non-trivial task of assessing the numerical dosimetry on realistic anatomical models with different postures resembling those of a driver or a bystander. This is therefore a valuable combination of an ad-hoc formulation, together with a consolidated software tool, which made it possible for the first time to account for all electro-geometrical difficulties coming both from the modeling of a realistic vehicle and a human body.

\section{Materials and Methods}

\subsection{Car Modeling}

The compact vehicle considered in this paper is a FIAT 500 car. A model suitable for electromagnetic computations is not so easy to identify. To give an example, the commercial CAD available at https://hum3d.com/3d-models/fiat-500/ (accessed on 7 February 2021) includes many details such as the license plate, some internal components, the logo of the CAD provider, and so on (see Figure 1a), which are not necessary for simulation purposes. In addition, these models are created for rendering purposes, and objects are represented either by surfaces or by volumes. This fact makes the generation of a good mesh very hard, if not impossible. For this reason, a brand new CAD was developed keeping in mind that it has to be used for numerical electromagnetic simulations (see Figure 1b). This model, together with other CAD models of vehicles, can be freely obtained at the following link https://github.com/cadema-PoliTO/vehicle4em (accessed on 7 February 2021).

In this paper, only the chassis of the car is considered and modeled as a surface mesh (see Figure 1c), since the numerical formulation is based on the SIBC method [11]. In order to investigate the effect of the car body shielding on the exposure assessment, two different materials were considered: aluminum and carbon fiber. The former represents the currently adopted material in compact vehicles, while the latter could be employed in future EVs. A conductivity of $\sigma=3 \times 10^{7} \mathrm{~S} / \mathrm{m}$ was used for the aluminum, whereas a homogeneous conductivity of $\sigma=4 \times 10^{4} \mathrm{~S} / \mathrm{m}$ was employed for the CF, as derived in [15]. 


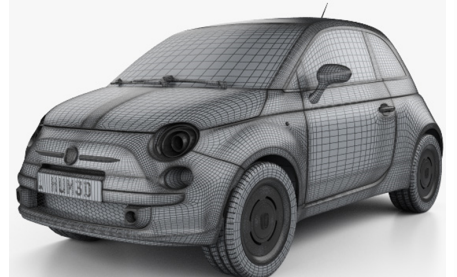

(a)

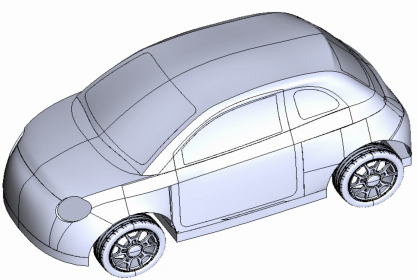

(b)

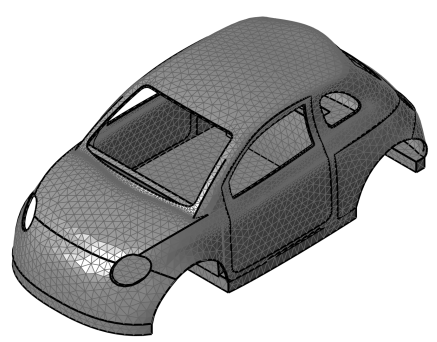

(c)

Figure 1. Commercial CAD model of a FIAT 500 (a), simplified CAD model for electromagnetic simulations (b), and the surface mesh used in this paper (c).

\subsection{WPT System Configuration}

Recently, several standards for WPT applications in EVs have been established. Among these, the International Electrotechnical Commission (IEC) and the Society of Automotive Engineers (SAE) are the most adopted $[16,17]$. They mainly specify the transfer power and the operational frequency of the WPT system, together with other parameters and specifications. For a compact car, as the considered one, the input power is set to $7.7 \mathrm{kVA}$, while the operational frequency is fixed to $85 \mathrm{kHz}$. The clearance between the receiving coil and the ground is set to $200 \mathrm{~mm}$, corresponding to the WPT2/Z3 configuration described in the Recommended Practice SAE J2954 [17]. Each coil is made of 8 turns, and the current flowing into a single-turn is $26 \mathrm{~A}$ for the transmitter and j26 A for the receiver. Both coils are shielded by two thin layers of aluminum and ferrite with an outer dimension of approximately $420 \times 420 \mathrm{~mm}^{2}$, as shown in Figure 2 .

In order to investigate the worst exposure scenario, both the case of perfect alignment (see Figure 2a,b) and of maximum misalignment, as suggested by SAE J2954 [17], i.e., $d_{x}=-75 \mathrm{~mm}$ and $d_{y}=100 \mathrm{~mm}$ (see Figure 2c,d), were considered.

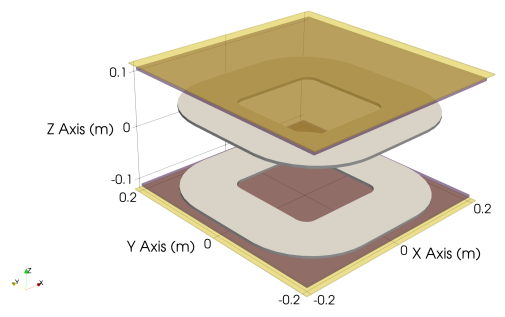

(a)

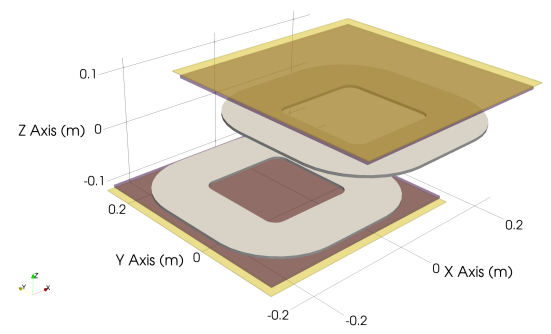

(c)

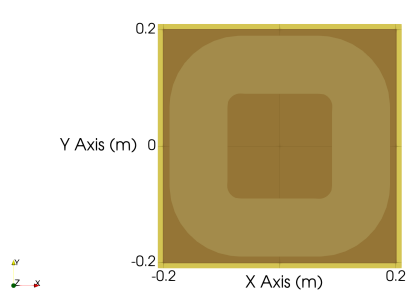

(b)

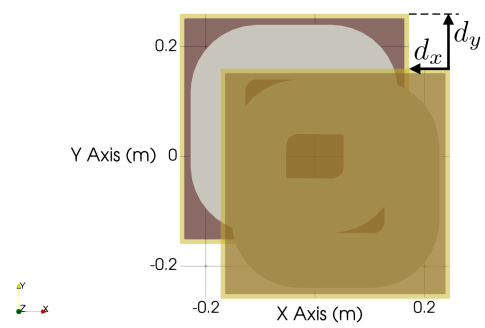

(d)

Figure 2. WPT2/Z3 system with perfect alignment $(\mathbf{a}, \mathbf{b})$ and maximum misalignment $(\mathbf{c}, \mathbf{d})$.

\subsection{Exposure Scenarios for Numerical Dosimetry}

The exposure scenarios consist of two realistic anatomical models placed nearby or inside the car, as illustrated in Figure 3. Specifically, Duke and Ella from the Virtual Population ViP 3.0 (https:/ /itis.swiss/virtual-population accessed on 7 February 2021) were employed. The former is in a driving position at a distance $t_{z}=30 \mathrm{~cm}$ from the 
secondary coil, while the latter is in a standing posture outside the car at a distance $t_{y}=22.3 \mathrm{~cm}$ from the primary coil in the misaligned position (see Figure $3 \mathrm{~b}$ ).

Tissue dielectric properties were assigned from the IT'ISdatabase [18], with the exception of the skin where a higher conductivity value was adopted, as described in $[19,20]$. A uniform grid size of $2 \mathrm{~mm}$ was used to discretize the computational domain embedding the anatomical models (see Figure 3a), yielding $430 \times 210 \times 520$ cells for Ella and $270 \times 150 \times 900$ cells for Duke. Despite these large cell numbers, numerical dosimetry simulations took only a few minutes on a machine with an Intel CPU X5650 (12 cores/ 24 threads at $2.67 \mathrm{GHz}$ ).

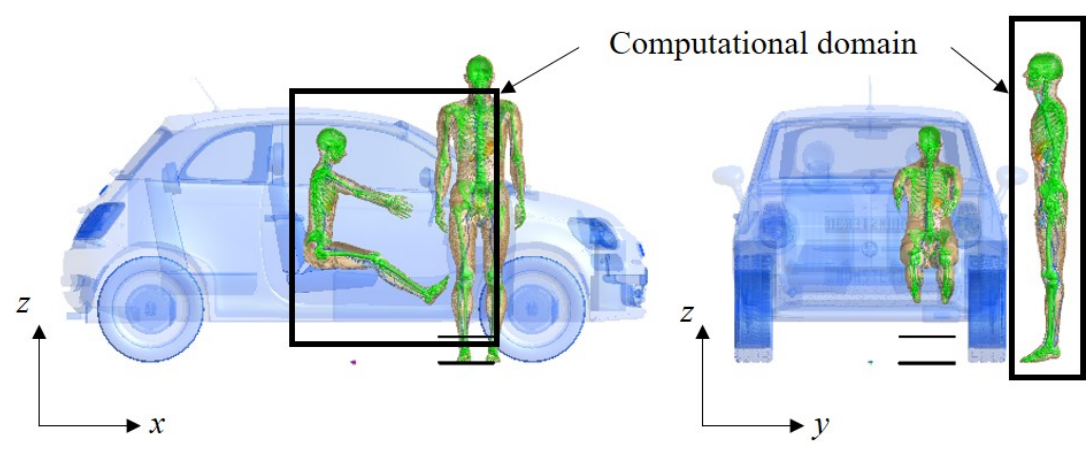

(a)
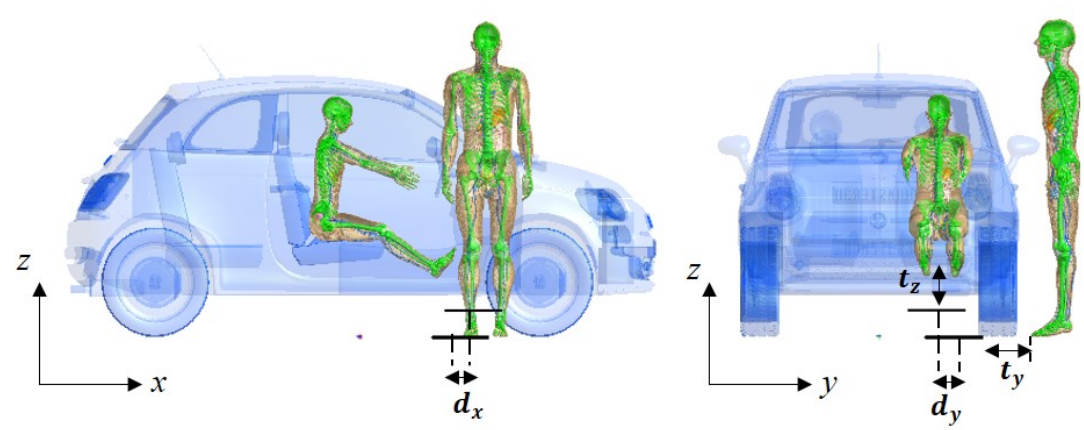

(b)

Figure 3. Exposure scenarios for the aligned (a) and misaligned (b) coils.

\subsection{Magnetic Field Evaluation}

The evaluation of the magnetic field coming from the WPT system is a challenging task. Indeed, the problem is characterized by a limited active region made by the conductive and magnetic parts and the conductors, while the boundary of the problem is unlimited. In addition, the geometrical dimensions of the car are much larger than the thickness of the sheet metal of the chassis. This fact is also emphasized by the small skin depth of the induced eddy currents. Such unbounded multiscale problems are not easily solved using standard volume-based methods like finite elements. For this reason, a hybrid BEM/SIBC formulation was used [11]. The BEM accounts for the unbounded air domain and for the non-conductive magnetic materials, whereas the SIBC is used to model the eddy currents in thin conductive layers. The SIBC is here described in terms of integral variables, which is typical of the algebraic formulations [21]. The magnetic flux density (B-field) is then evaluated in a post-processing stage inside the box regions embedding the anatomical models (see Figure 3a), which are not considered in this stage since they do not perturb the magnetic field at such frequencies, as demonstrated in [22]. 
BEM equations, topological equations, constitutive equations, and continuity equations are coupled to obtain the final system:

$$
\left[\begin{array}{cc}
-\mathbf{H} & \mathbf{W} \\
\mathbf{C Z C}^{\mathrm{T}} & \mathrm{j} \omega \mu_{0} \mathbf{S}
\end{array}\right]\left[\begin{array}{c}
\boldsymbol{\psi} \\
\partial_{n} \boldsymbol{\psi}
\end{array}\right]=\left[\begin{array}{c}
\mathbf{0} \\
\mathbf{b}
\end{array}\right]
$$

with $\mu_{0}$ the permeability of the air, $\omega$ the angular frequency, $\mathbf{H}$ and $\mathbf{W}$ the standard matrices of the BEM formulation, $\psi$ and $\partial_{n} \psi$ the reduced magnetic scalar potential and its normal derivative, $\mathbf{Z}$ the impedance matrix that links current to voltages, $\mathbf{S}$ the matrix with triangle surfaces, and $\mathbf{C}$ the discrete curl operator. The right-hand side is defined as $\mathbf{b}=\mathbf{C Z} \mathbf{h}_{\mathrm{S}}+\mathrm{j} \omega \mu_{0} \mathbf{S} \mathbf{H}_{\mathrm{Sn}}$, where $\mathbf{h}_{\mathrm{S}}$ is the magnetic field created by the source currents on the primal edges and $\mathbf{H}_{S n}$ is the orthogonal components of the same magnetic field on the triangles.

For naive problems, direct solvers can be used to solve (1). However, this approach becomes impractical for medium-scale problems, because the lower-left block requires the preliminary calculation of the impedance matrix $\mathbf{Z}=\mathbf{Y}^{-1}$. The explicit inversion of the admittance matrix is computationally intensive and gives rise to a dense block in addition to the BEM matrices $\mathbf{H}$ and $\mathbf{W}$ [11].

For nontrivial problems, the complex nonsymmetric indefinite system is solved by an iterative Krylov subspace solver like GMRES [23]. With the use of iterative solvers, the effective availability of the matrix in (1) is not strictly necessary, but only the matrix vector product is required. To this aim, the effective calculation of $\mathbf{Z}$ can be avoided in favor of a more efficient LU factorization.

As suggested in [24], the spectral properties of (1) can be further improved by calculating the Schur complement of the lower-right block $j \omega \mu_{0} \mathbf{S}$ and solving with respect to $\partial_{n} \psi$ :

$$
\partial_{n} \boldsymbol{\psi}=-\mathbf{H}_{S n}+\left(\mathrm{j} \omega \mu_{0} \mathbf{S}\right)^{-1}\left(\mathbf{C Z C}^{T} \boldsymbol{\psi}-\mathbf{C Z h}_{S}\right)
$$

Substituting (2) in (1) yields:

$$
\left[\mathbf{H}+\mathbf{W}\left(\mathrm{j} \omega \mu_{0} \mathbf{S}\right)^{-1} \mathbf{C Z C} \mathbf{C}^{\mathrm{T}}\right] \boldsymbol{\psi}=\mathbf{b}_{\mathrm{s}}
$$

with $\mathbf{b}_{\mathrm{S}}=\mathbf{W}\left(\mathbf{j} \omega \mu_{0} \mathbf{S}\right)^{-1} \mathbf{C Z} \mathbf{h}_{\mathrm{S}}+\mathbf{W} \mathbf{H}_{\mathrm{S} n}$.

Because the matrix $\mathbf{S}$ is diagonal, the calculation of the Schur complement is not computationally intensive. Again, the use of GMRES does not require the effective assembling of $\mathbf{H}+\mathbf{W}\left(\mathrm{j} \omega \mu_{0} \mathbf{S}\right)^{-1} \mathbf{C Z C} \mathbf{C}^{\mathrm{T}}$, but only the supply of the matrix-vector product $\mathbf{H} \psi+\mathbf{W}\left(\mathrm{j} \omega \mu_{0} \mathbf{S}\right)^{-1} \mathbf{C Z C}^{\mathrm{T}} \boldsymbol{\psi}$ to the solver.

All simulations of the WPT system taking into account the car body shielding effect were carried out on a machine with an Intel CPU i9-7900X (10 cores/20 threads at $4.3 \mathrm{GHz}$ ). In Equation (3), the number of unknowns corresponds to the number of faces of the mesh that, in this paper, is 70,132 (36,926 for the WPT system and 33,206 for the car body). GMRES iterations are stopped when the relative error $(\|A x-b\| /\|b\|)$ is lower than $10^{-10}$. Figure 4 shows the convergence of the GMRES solver when the coils are perfectly aligned in the case of car body made of aluminum or carbon fiber. The lower conductivity of carbon fiber makes the convergence slower, but does not preclude reaching the desired relative error. Regarding the computational time, the convergence is reached in about $7 \mathrm{~h}$ for the aluminum chassis and $15 \mathrm{~h}$ for the carbon fiber chassis. 


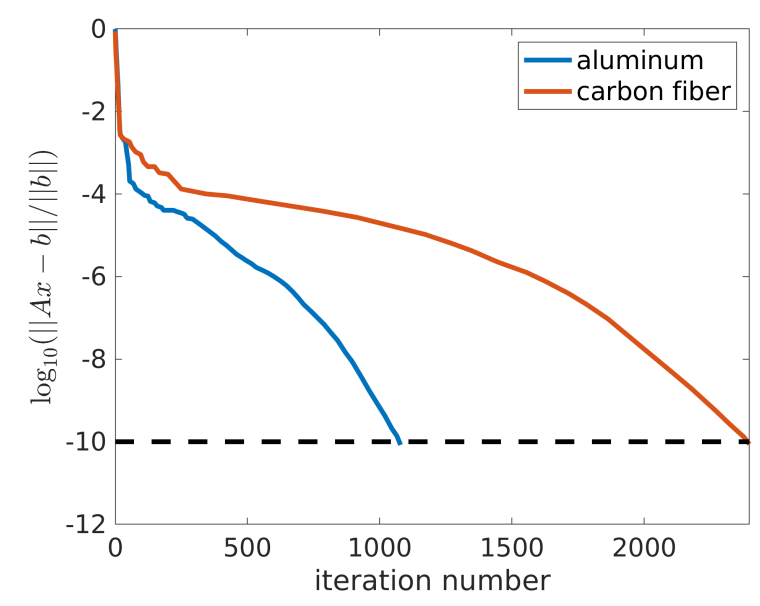

Figure 4. Convergence of the GMRES solver when the coils are perfectly aligned in the case of a car body made of aluminum (blue) or carbon fiber (red).

\subsection{LF Dosimetry}

When considering LF dosimetry, the SPFD method is often the preferred one because the induced currents in the human body do not modify the source field, and the problem can be formulated with the scalar potential as nodal unknowns [9]. Using the algebraic framework, the SPFD is given by [13]:

$$
\mathbf{G}^{\mathrm{T}} \mathbf{M}_{\sigma} \mathbf{G} \boldsymbol{\varphi}=-\mathbf{j} \omega \mathbf{G}^{\mathrm{T}} \mathbf{M}_{\sigma} \mathbf{a}_{\mathrm{s}}
$$

where, for a voxel-based human model, $\mathbf{M}_{\sigma}$ is a diagonal conductance matrix, $\mathbf{G}$ is the edge-to-node incidence matrix, $\mathbf{a}_{\mathrm{s}}$ is the line integral of the magnetic vector potential due to the sources, and $\varphi$ is the electric scalar potential [13].

The magnetic vector potential (A-field) is not readily accessible from the hybrid formulation. This issue can be solved by using a curl-inversion procedure, which was extensively described in [12-14]. In this paper, we tested the commercial software Sim4Life, which implemented the curl-inversion procedure based on a previous work of the authors [12]. For the sake of completeness, the equations for the curl-inversion are given:

$$
\begin{aligned}
A_{x}= & -\int_{0}^{y}\left(\frac{1}{3} B_{z}\left(x, y^{\prime}, z\right)+\frac{1}{6} B_{z}\left(x, y^{\prime}, 0\right)\right) d y^{\prime} \\
& +\int_{0}^{z}\left(\frac{1}{3} B_{y}\left(x, y, z^{\prime}\right)+\frac{1}{6} B_{y}\left(x, 0, z^{\prime}\right)\right) d z^{\prime} \\
A_{y}= & -\int_{0}^{z}\left(\frac{1}{3} B_{x}\left(x, y, z^{\prime}\right)+\frac{1}{6} B_{x}\left(0, y, z^{\prime}\right)\right) d z^{\prime} \\
& +\int_{0}^{x}\left(\frac{1}{3} B_{z}\left(x^{\prime}, y, z\right)+\frac{1}{6} B_{z}\left(x^{\prime}, y, 0\right)\right) d x^{\prime} \\
A_{z}= & -\int_{0}^{x}\left(\frac{1}{3} B_{y}\left(x^{\prime}, y, z\right)+\frac{1}{6} B_{y}\left(x^{\prime}, 0, z\right)\right) d x^{\prime} \\
& +\int_{0}^{y}\left(\frac{1}{3} B_{x}\left(x, y^{\prime}, z\right)+\frac{1}{6} B_{x}\left(0, y^{\prime}, z\right)\right) d y^{\prime}
\end{aligned}
$$

With reference to Figure 5, the numerical model of the source is used to compute the B-field at discrete points in a bounded hexahedral grid, and Equation (5) is then used to get the A-field at the same points. This A-field distribution is interpolated on the human model, and eventually, the electric scalar potential is obtained by solving Equation (4). It is worth stressing that Figure 5 is purely descriptive, and more points were used in the actual simulations. The relative error introduced in the solution is shown to be negligible for a discretization of the bounded volume in the order of centimeters [14]. 


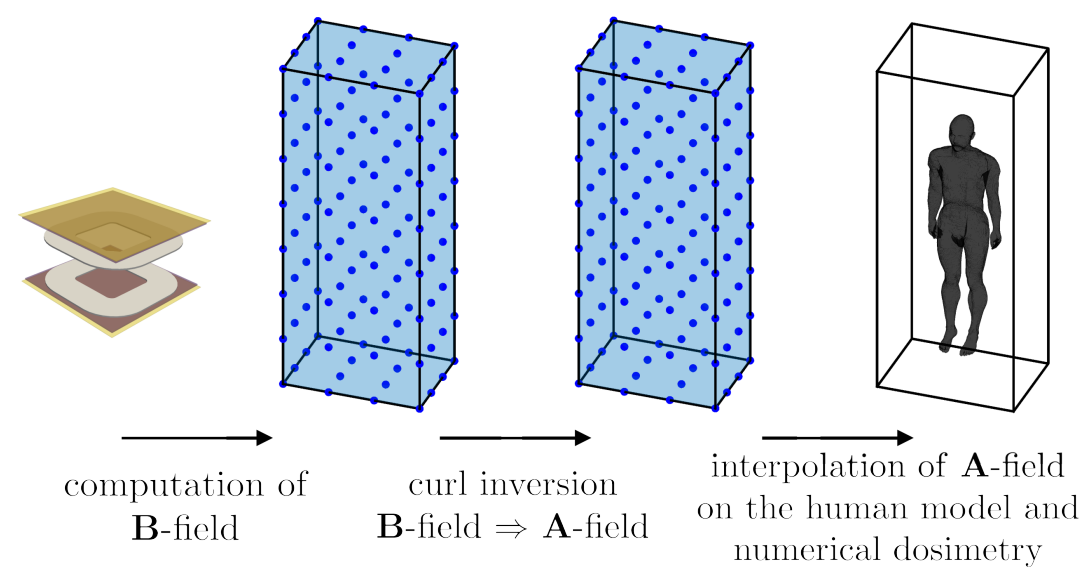

Figure 5. The human model is bounded in a hexahedral grid. Knowing the B-field at the blue points, it is possible to define a compatible A-field that, after interpolation, is used as the source term in Equation (4).

Finally, by knowing the electric scalar potential $\varphi$, it is straightforward to compute the induced electric field in the human body as required by the dosimetric assessment.

\section{Numerical Dosimetry Results}

The exposure assessment of the investigated WPT system against the EMF limits for the general public provided by the International Commission on Non-Ionizing Radiation Protection (ICNIRP) is hereby provided [25]. First, the magnetic flux density computed outside or inside the car is determined without the presence of the human models for comparison with the reference level (RL). Then, the electric field induced inside the human body is evaluated for comparison with the basic restriction (BR).

\subsection{RL Numerical Dosimetry}

Figure $6 a, b$ depicts the magnetic field distributions inside the computational domain when considering an $\mathrm{Al}$ chassis for both aligned and misaligned coil configurations, respectively. The same is reported in Figure 7a,b for the CF chassis. In these figures, the anatomical models are overlaid on the exposure scenario only for the sake of clarity, i.e., to facilitate the understanding of the compliance. Indeed, the green areas are the region where the RL (i.e., $B_{\text {lim }}=27 \mu \mathrm{T}$ ) is exceeded. As can be observed, the ICNIRP-RL is never exceeded in Duke standing outside for both chassis materials (almost exceeding for the misaligned case). Instead, it is barely exceeded in the heel of Ella driving in the car body made of aluminum, while it is largely exceeded in the leg and feet areas for the CF chassis. Thus, compliance against BR is needed in these latter cases. 


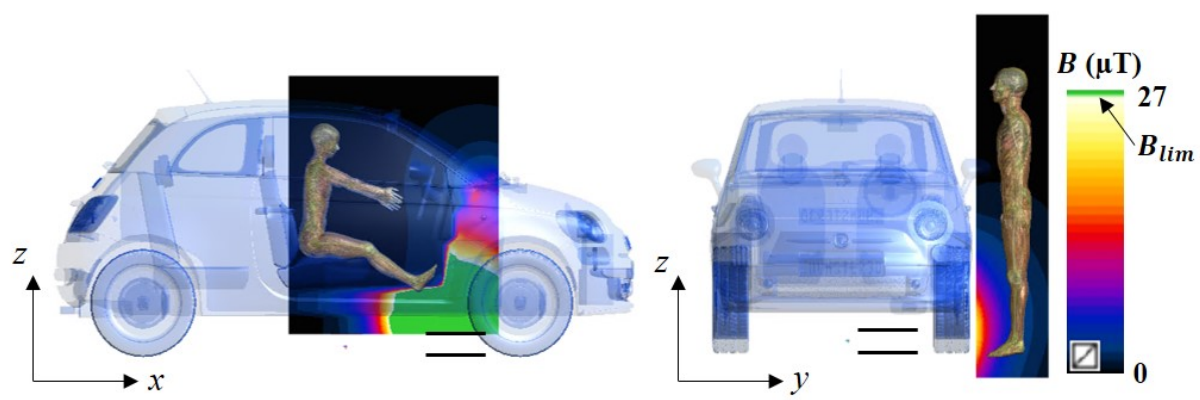

(a)
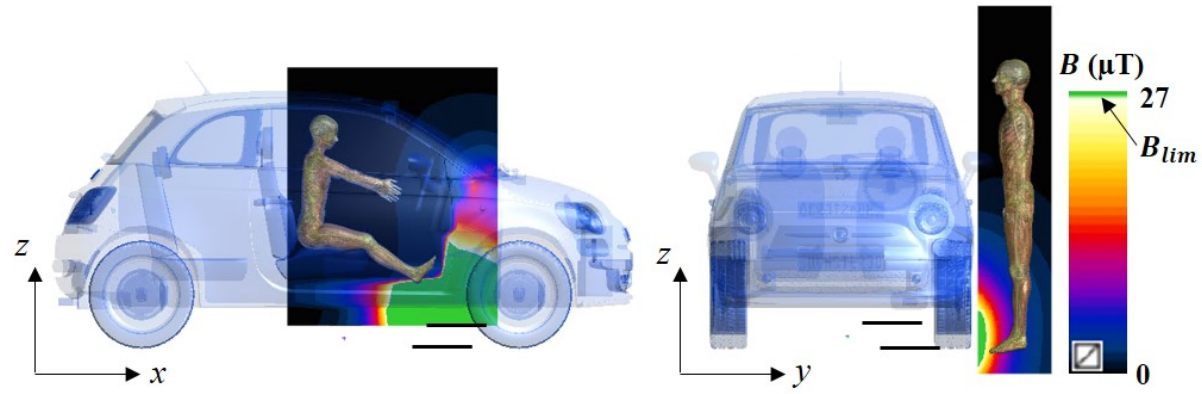

(b)

Figure 6. B-field distributions for the aligned (a) and misaligned (b) coil positions with the $\mathrm{Al}$ chassis.
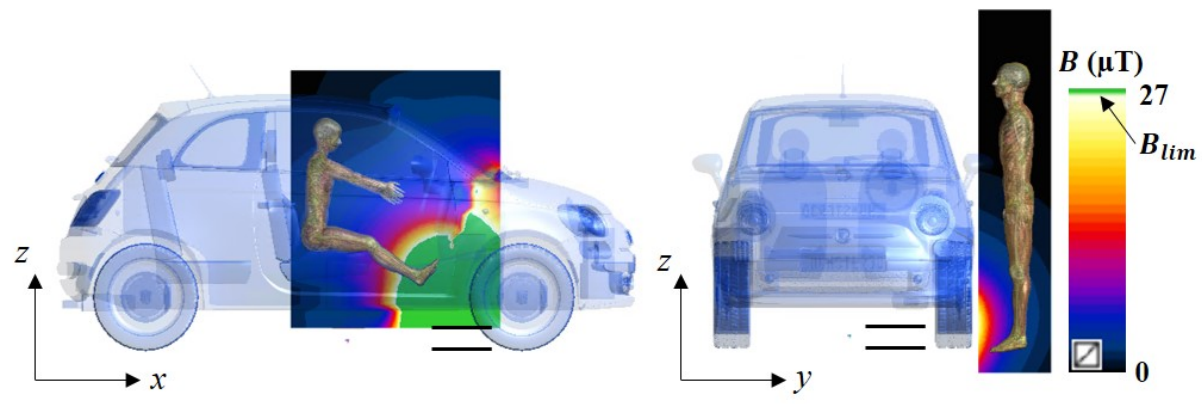

(a)
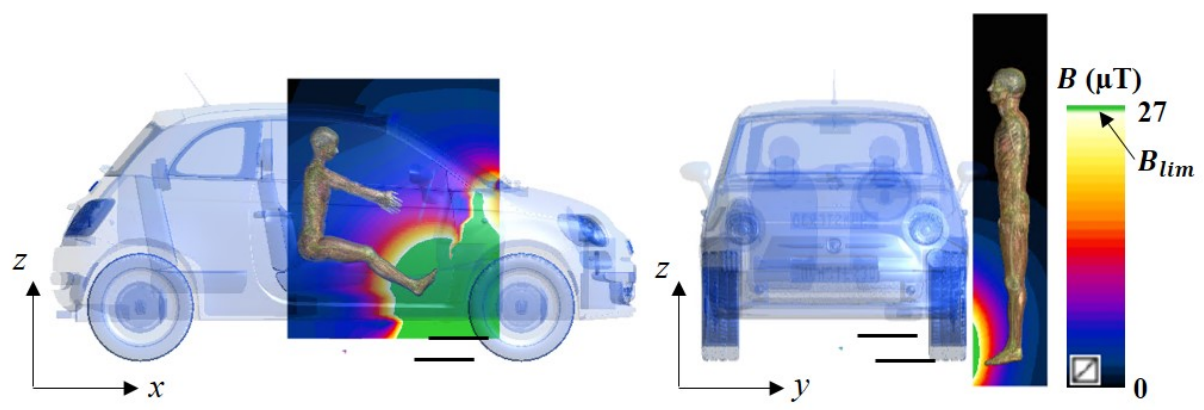

(b)

Figure 7. B-field distributions for the aligned (a) and misaligned (b) coil positions with the carbon fiber $(\mathrm{CF})$ chassis.

\subsection{BR Numerical Dosimetry}

The induced electric field distributions inside the two anatomical models are reported in Figure 8 for both chassis materials and both coil positions, respectively. The obtained results show that the ICNIRP-BR is never exceeded, except for a small portion of the feet in the driving passenger when the CF chassis is considered. However, it is worth 
noting that ICNIRP suggests determining compliance against a $2 \times 2 \times 2 \mathrm{~mm}^{3}$ volume averaging and the 99-th percentile of the peak induced electric field [25]. In this paper, we use anatomical models with a voxel resolution of $2 \mathrm{~mm}$ that corresponds to the averaging volume proposed by the ICNIRP; therefore, only the 99-th percentile has to be computed. For the sake of completeness, also the 99.9-th percentile is hereby evaluated since the 99-th percentile has been sometimes determined to underestimate the compliance, especially in the case of non-uniform/localized exposure [8,26-30].

To better quantify these results, the values of the exposure assessment are summarized in Table 1, where $E_{\max }$ is the peak induced electric field, whereas $E_{99.9}$ and $E_{99}$ are the 99.9-th and 99-th percentiles, respectively. As can be observed, when comparing the latter with the $\mathrm{BR}$, the overexposure is always negative (at least $-16 \mathrm{~dB}$ ), meaning that the considered exposure scenario is far away from exceeding the ICNIRP-BR. The influence of the chassis material is also evident, with an average shielding of 3-4 dB on the induced electric fields for the $\mathrm{Al}$ compared to the $\mathrm{CF}$.
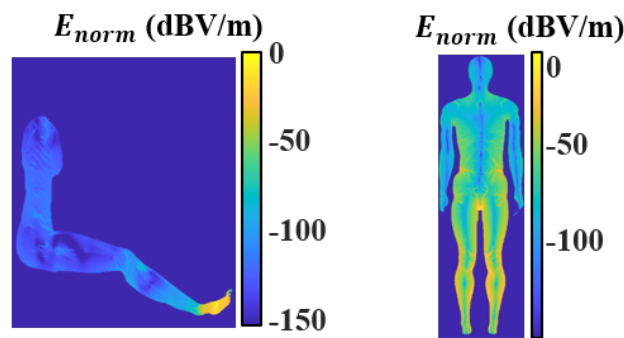

(a)
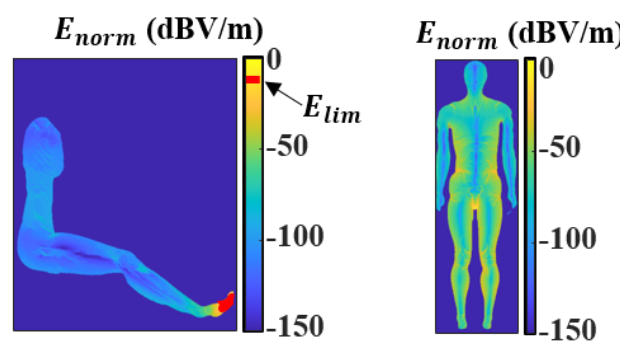

(c)
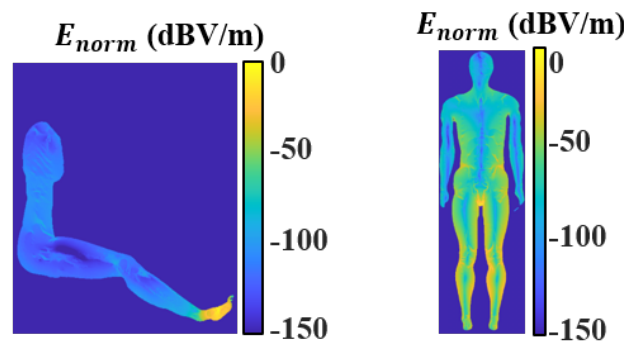

(b)
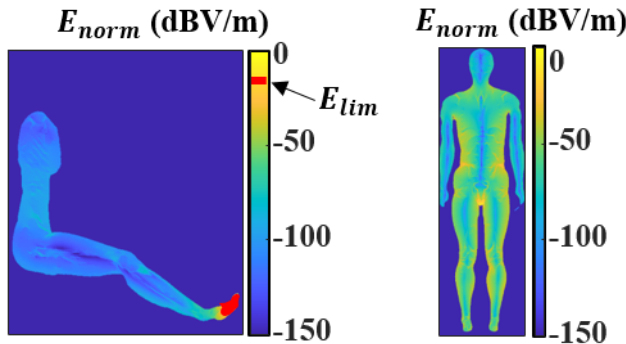

(d)

Figure 8. Induced electric field distributions inside Ella (left) and Duke (right) for the aligned (a) and misaligned (b) coil positions in the Al chassis and for the aligned (c) and misaligned (d) coil positions in the CF chassis. $E_{\text {norm }}$ is the logarithmic electric field normalized to the peak value. $E_{l i m}$ is the basic restriction $(B R)=11.48 \mathrm{~V} / \mathrm{m}$ (the red area is the portion where the $\mathrm{BR}$ is exceeded).

Table 1. Summary of the compliance assessment with the BR for the considered exposure scenarios.

\begin{tabular}{l|c|cccc}
\hline $\begin{array}{l}\text { Exposure } \\
\text { Scenario }\end{array}$ & $\begin{array}{c}\text { Chassis } \\
\text { Material }\end{array}$ & $\begin{array}{c}\boldsymbol{E}_{\max } \\
(\mathbf{V} / \mathbf{m})\end{array}$ & $\begin{array}{c}\boldsymbol{E}_{99.9} \\
\mathbf{( V / m})\end{array}$ & $\begin{array}{c}\boldsymbol{E}_{99} \\
(\mathbf{V} / \mathbf{m})\end{array}$ & $\begin{array}{c}\text { Overexposure } \\
(\mathbf{d B})\end{array}$ \\
\hline Ella-Aligned & & 8.26 & 1.84 & 0.71 & -18.48 \\
Ella-Misaligned & aluminum & 7.69 & 1.36 & 0.57 & -19.22 \\
Duke-Aligned & & 0.27 & 0.11 & 0.07 & -44.11 \\
Duke-Misaligned & & 0.53 & 0.25 & 0.12 & -39.56 \\
\hline Ella-Aligned & & 19.21 & 5.86 & 1.71 & -16.48 \\
Ella-Misaligned & \multirow{2}{*}{ carbon fiber } & 24.00 & 6.94 & 1.76 & -16.23 \\
Duke-Aligned & & 0.76 & 0.36 & 0.14 & -38.11 \\
Duke-Misaligned & & 0.90 & 0.44 & 0.17 & -36.45 \\
\hline
\end{tabular}




\section{Conclusions}

In this paper, the human exposure to a WPT system aimed to recharge a compact vehicle was studied. The analysis was performed selecting suitable and separate tools to analyze the magnetic field source and to evaluate the induced electric fields. The magnetic field source was handled by an ad-hoc software based on a hybrid scheme, whereas the numerical dosimetry was carried out using a commercial software. The proposed approach was able to handle the complex shape of the compact vehicle (namely a FIAT 500) and anatomical models in a seamless way.

In order to investigate the effect of the car body shielding on the exposure assessment, two different materials were considered for the vehicle chassis: aluminum and carbon fiber. The former represents the currently adopted material in compact vehicles, whereas the latter should be employed more often in future EVs. Although hybrid solutions could most likely be used, these two materials therefore cover the upper and lower band of the conductivity values that an EV cabin can assume.

The proposed method was tested on the case of a WPT system mounted close to the left-front wheel. The exposure was evaluated both for the driver and a bystander. Aligned and misaligned coil configurations of the WPT system were considered as well. From the obtained results, it is shown that the considered cases are not compliant with the reference level, especially for the driver position within a car body frame made of carbon fiber. Instead, numerical dosimetry confirmed that the basic restriction is never exceeded, at least for the considered scenarios.

Finally, it should be noted that the exposure results are affected by many factors, first and foremost by the WPT geometry and coil currents. Different charging spot layouts or different charging technologies may result in different magnetic field distribution and, in turn, different induced electric fields. However, the methodology proposed in this paper is general and can be easily extended to new devices or charging systems.

Author Contributions: The authors contributed equally to this work. All authors read and agreed to the published version of the manuscript.

Funding: This research received no external funding.

Institutional Review Board Statement: Not applicable.

Informed Consent Statement: Not applicable.

Data Availability Statement: Not applicable.

Acknowledgments: The authors would like to thank Donato Manesi (Politecnico di Torino) for the support given in the CAD modeling and Francesco Di Blasio and Gabriella Febbo (University of L'Aquila) for their valuable support with the dosimetric simulations.

Conflicts of Interest: The authors declare no conflict of interest.

\section{References}

1. International Energy Agency (IEA). Global Energy and $\mathrm{CO}_{2}$ Status Report; IEA: Paris, France, 2018.

2. International Energy Agency (IEA). Global EV Outlook; IEA: Paris, France, 2019.

3. Machura, P.; De Santis, V.; Li, Q. Driving range of Electric Vehicles charged by Wireless Power Transfer. IEEE Trans. Veh. Technol. 2020, 69, 5968-5982. [CrossRef]

4. Romo, J.; Cañibano, E.; Merino, J.C. Lightweighting and passive safety for urban electric vehicle. In Proceedings of the 2017 Electric Vehicles International Conference (EV), Bucharest, Romania, 5-6 October 2017; pp. 1-5. [CrossRef]

5. Shimamoto, T.; Laakso, I.; Hirata, A. In-situ electric field in human body model in different postures for wireless power transfer system in an electrical vehicle. Phys. Med. Biol. 2015, 60, 163-173. [CrossRef]

6. Cirimele, V.; Freschi, F.; Giaccone, L.; Pichon, L.; Repetto, M. Human exposure assessment in Dynamic Inductive Power Transfer for automotive applications. IEEE Trans. Magn. 2017, 53, 1-4. [CrossRef]

7. De Santis, V.; Campi, T.; Cruciani, S.; Laakso, I.; Feliziani, M. Assessment of the induced electric fields in a Carbon-Fiber Electrical Vehicle equipped with a Wireless Power Transfer system. Energies 2018, 11, 684. [CrossRef] 
8. Arduino, A.; Bottauscio, O.; Chiampi, M.; Giaccone, L.; Liorni, I.; Kuster, N.; Zilberti, L.; Zucca, M. Accuracy assessment of numerical dosimetry for the evaluation of human exposure to Electric Vehicle inductive charging systems. IEEE Trans. Electromag. Compat. 2020, 62, 1939-1950. [CrossRef]

9. Dawson, T.; Stuchly, M. Analytic validation of a three-dimensional scalar-potential finite-difference code for low-frequency magnetic induction. Appl. Comput. Electrom. 1996, 11, 72-81. ISSN: 1054-4887.

10. Zang, M.; Cimala, C.; Clemens, M.; Dutiné, J.; Timm, T.; Schmuelling, B. A Co-Simulation Scalar-Potential Finite Difference Method for the Numerical Analysis of Human Exposure to Magneto-Quasi-Static Fields. IEEE Trans. Magn. 2017, 53, 1-4. [CrossRef]

11. Freschi, F.; Giaccone, L.; Repetto, M. Algebraic formulation of nonlinear surface impedance boundary condition coupled with BEM for unstructured meshes. Eng. Anal. Bound. Elem. 2018, 88, 104-114. [CrossRef]

12. Laakso, I.; De Santis, V.; Cruciani, S.; Campi, T.; Feliziani, M. Modelling of induced electric fields based on incompletely known magnetic fields. Phys. Med. Biol. 2017, 62, 6567. [CrossRef]

13. Freschi, F.; Giaccone, L.; Cirimele, V.; Canova, A. Numerical assessment of low-frequency dosimetry from sampled magnetic fields. Phys. Med. Biol. 2018, 63, 015029. [CrossRef]

14. Conchin Gubernati, A.; Freschi, F.; Giaccone, L.; Campi, T.; De Santis, V.; Laakso, I. Comparison of numerical techniques for the evaluation of human exposure from measurement data. IEEE Trans. Magn. 2019, 55, 1-4. [CrossRef]

15. Campi, T.; Cruciani, S.; De Santis, V.; Maradei, F.; Feliziani, M. Magnetic field behavior in a carbon-fiber electrical vehicle charged by a wireless power transfer system. In Proceedings of the 2017 International Symposium on Electromagnetic Compatibility-EMC EUROPE, Angers, France, 4-7 September 2017; pp. 1-6. [CrossRef]

16. IEC 61980-1. Electric Vehicle Wireless Power Transfer (WPT) Systems-Part 1: General Requirements; IEC: Geneva, Switzerland, 2015.

17. International SAE J2954-Wireless Power Transfer for Light-Duty Plug-In/Electric Vehicles and Alignment Methodology; SAE: Warrendale, PA, USA, 2019.

18. Hasgall, P.A.; Di Gennaro, F.; Baumgartner, C.; Neufeld, E.; Lloyd, B.; Gosselin, M.; Payne, D.; Klingenböck, A.; Kuster, N. IT'IS Database for Thermal and Electromagnetic Parameters of Biological Tissues. 2018. Available online: www.itis.swiss/database (accessed on 12 December 2020).

19. De Santis, V.; Chen, X.L.; Laakso, I.; Hirata, A. An equivalent skin conductivity model for low-frequency magnetic field dosimetry. Biomed. Phys. Eng. Express 2015, 1, 1-10. [CrossRef]

20. De Santis, V.; Chen, X.L.; Cruciani, S.; Campi, T.; Feliziani, M. A novel homogenization procedure to model the skin layers in LF numerical dosimetry. Phys. Med. Biol. 2016, 61, 4402-4411. [CrossRef]

21. Tonti, E. A direct discrete formulation of field laws: The cell method. Comput. Model. Eng. Sci. 2001, 2, 237-258. [CrossRef]

22. Hirata, A.; Ito, F.; Laakso, I. Confirmation of quasi-static approximation in SAR evaluation for a wireless power transfer system. Phys. Med. Biol. 2013, 58, N241-N249. [CrossRef]

23. Kim, M.; Yun, I. An efficient implementation of the generalized minimum residual algorithm with a new preconditioner for the boundary element method. Eng. Anal. Bound. Elem. 2011, 35, 1214-1224. [CrossRef]

24. Mandel, J. On block diagonal and Schur complement preconditioning. Numer. Math. 1990, 58, 79-93. [CrossRef]

25. ICNIRP. Guidelines for Limiting Exposure to Time-Varying Electric and Magnetic Fields (1 Hz-100 kHz). Health Phys. 2010, 99, 818-836. [CrossRef]

26. Kos, B.; Valič, B.; Miklavčič, D.; Kotnik, T.; Gajšek, P. Pre- and post-natal exposure of children to EMF generated by domestic induction cookers. Phys. Med. Biol. 2011, 56, 6149-6160. [CrossRef]

27. Laakso, I.; Hirata, A. Reducing the staircasing error in computational dosimetry of low-frequency electromagnetic fields. Phys. Med. Biol. 2012, 57, 25-34. [CrossRef] [PubMed]

28. De Santis, V.; Chen, X.L. On the issues related to compliance assessment of ICNIRP 2010 basic restrictions. J. Rad. Prot. 2014, 34, N31. [CrossRef] [PubMed]

29. Diao, Y.; Gomez-Tames, J.; Rashed, E.A.; Kavet, R.; Hirata, A. Spatial averaging schemes of in situ electric field for low-frequency magnetic field Eexposures. IEEE Access 2019, 7, 184320-184331. [CrossRef]

30. Giaccone, L. Compliance of non-sinusoidal or pulsed magnetic fields generated by industrial sources with reference to human exposure guidelines. In Proceedings of the 2020 International Symposium on Electromagnetic Compatibility-EMC EUROPE, Rome, Italy, 23-25 September 2020; pp. 1-6. [CrossRef] 